The Astrophysical Journal, $171: 71-77,1972$ January 1

(c) 1972. The University of Chicago. All rights reserved Printed in U S A

\title{
THE LITHIUM ISOTOPE RATIO IN F AND G FIELD STARS
}

\author{
Judith Gamora Cohen* \\ Hale Observatories \\ California Institute of Technology, Carnegie Institution of Washington \\ Received 1971 June 21; revised 1971 August 16
}

\begin{abstract}
A Fabry-Perot interferometer was used to obtain high-resolution profiles of the resonance line of $\mathrm{Li} \mathrm{I}$ and $\lambda 6717.69$ of $\mathrm{Ca} \mathrm{I}$ in 14 bright $\mathrm{F}, \mathrm{G}$, and early $\mathrm{K}$ field stars. The observational data were fitted by computed theoretical line profiles to determine the rotational velocity, $\mathrm{Li}$ abundance, and $\mathrm{Li}$ isotope ratio for each star. The ${ }^{6} \mathrm{Li} /{ }^{7} \mathrm{Li}$ ratio was 0.1 or less in all the program stars.
\end{abstract}

\section{INTRODUCTION}

Valuable information on nuclear and chemical processes which may occur during the lifetime of a star can be obtained from the lithium abundance and isotope ratio. The lithium abundance for stars of various spectral types has been extensively studied (Wallerstein and Conti 1969), and the resulting correlations are at least partially understood. An attempt to define the $\mathrm{Li}$ isotope ratio has previously been carried out on a limited number of stars by Herbig (1964), who used photographic spectra with a dispersion of $4.1 \AA \mathrm{mm}^{-1}$. Since Herbig could not resolve the profile of the $\mathrm{Li}$ line, which is a blend of several components, he tried to obtain the $\mathrm{Li}$ isotope ratio by measuring with a comparator the center-of-gravity wavelength $\left(\lambda_{1 / 2}\right)$ for the Li resonance line. In order to verify his results, a high-resolution Fabry-Perot interferometer was used to observe the $\mathrm{Li}$ resonance line in a group of field stars.

The Fabry-Perot interferometer (abbreviated FP) was mounted behind the focus of the 114-inch camera of the 100-inch telescope on Mount Wilson. A set of flats coated for $\mathrm{H} \alpha$ was used with a 1.13-mm spacer. The free range of the interferometer was $1.90 \AA$, and the measured full width of the instrumental profile at half-maximum was $0.091 \AA$. The exit slit of the coude scanner, which serves as the entrance slit of the FP, was set at $1.2 \AA$ to block all but one of the interference maxima. The instrumental arrangement for scanning in wavelength by varying the pressure $P$ in the FP while simultaneously rotating the grating is discussed in Vaughan (1967). (The grating position is denoted by "s.") A monitor channel to correct for seeing fluctuations (similar to the monitor of the coudé scanner described by Wilson 1968) counted photons in wide passbands on each side of the wavelength to which the FP was tuned. Both data and monitor channel have S-20 photomultipliers. At each wavelength point in the scan, an observation consisted of the count in the FP pulse counter when the monitor channel had reached a preset trigger level.

The neutral ${ }^{7} \mathrm{Li}$ resonance line at $6708 \AA$ is a fine-structure doublet, analogous to the D-lines of $\mathrm{Na} \mathrm{I}$, with components at 6707.76 and $6707.91 \AA$. The transition probabilities are in the ratio of $2: 1$, with $\lambda 6707.76$ being the stronger component. The ${ }^{6} \mathrm{Li}$ line has two components at 6707.92 and $6708.07 \AA$ with the same ratio of transition probabilities, so that the stronger ${ }^{6} \mathrm{Li}$ component coincides with the weaker ${ }^{7} \mathrm{Li}$ component. In most stars, no lines of $\mathrm{Li}$ other than the neutral resonance line are observed. From photographic spectra (19 spectra of 15 stars) at $4.1 \AA \mathrm{mm}^{-1}$, Herbig (1964) derived apparent

* Present address: Department of Astronomy, University of California, Berkeley

1 Using spectra of even lower dispersion, Conti (1969), Feast (1970), and others have used Herbig's method to derive apparent $\mathrm{Li}$ isotope ratios within the same range. 
isotope ratios (which we denote by ${ }^{6} \mathrm{Li} /{ }^{7} \mathrm{Li}$ ) of 0.0 to $0.5 .{ }^{1}$ The terrestrial and meteoritic ratio is 0.08 (Krankowsky and Müller 1967). We have observed profiles of the $\mathrm{Li} \mathbf{I}$ resonance line, $\lambda 6717.69$ of $\mathrm{Ca} \mathrm{I}$, and sometimes the D-lines of $\mathrm{Na} \mathrm{I}$ and $\lambda 6710.32$ of Fe $I$ in the program stars, which were selected to be sharp-lined stars bright enough to obtain interferometric line profiles in a reasonable length of time $\left(m_{V} \lesssim 5\right)$ and which have a strong resonance line of $\mathrm{Li}$ I. (The D-lines were observed with a different etalon coated for maximum reflectivity at $5890 \AA$.) All the stars studied by Herbig (1964) were observed, with the exception of three stars with ${ }^{6} \mathrm{Li} /{ }^{7} \mathrm{Li} \approx 0.0$, and $\iota \mathrm{Peg}$, a spectroscopic binary with a 10-day period and an amplitude of variation for $v_{r}$ of $90 \mathrm{~km} \mathrm{~s}^{-1}$.

\section{OBSERVATIONAL PROCEDURES}

Given a photographic spectrum of a star, the relative position of the $\mathrm{Li}$ line can be measured with respect to the many other stellar lines to derive an accurate wavelength scale near the $\mathrm{Li}$ feature. However, profiles of a very small number of lines are obtained when we observe with a FP. The interferometer is calibrated in $\lambda$ by scanning emission lines of a neon-filled $\mathrm{Li}$ hollow-cathode lamp which is used to illuminate a ground glass diffuser at the entrance slit to the spectrograph. Thus $\left(s_{0}, P_{0}\right)$ are known for $6707.76 \AA$ (the strongest component of the Li line) and for $6717.04 \AA$ (the central wavelength of a Ne I line). The quantities $\Delta P / \Delta \lambda$ and $\Delta s / \Delta \lambda$ can be determined from measurements with the calibration lamp, as well as calculated from properties of the instrument. Therefore, from the radial velocity $v_{r}$ of the star, plus a correction for orbital motions of the Earth, the $\left(s_{*}, P_{*}\right)$ which corresponds to 6707.76 and $6717.04 \AA$ in the rest frame of the star can be derived. In order to do this, $v_{r}$ must be known to within $\pm 0.5 \mathrm{~km} \mathrm{~s}^{-1}$, and it was not obvious that the radial velocities tabulated in the Yale Catalogue of Bright Stars (Hoffleit 1964) were sufficiently accurate. To determine $v_{r}$ more accurately, additional stellar lines such as $\lambda 6717.69$ of $\mathrm{Ca} \mathrm{I}$ were observed. The $v_{r}$ values from Hoffleit (1964), corrected for any shift between the observed and predicted central wavelengths of $\lambda 6717.69$ of $\mathrm{Ca} I$ and of the $\mathrm{D}$-lines, are those listed in Table 1.

Because the wavelength scale is critical in determining the isotope ratio, several tests have been performed to ensure that the instrument does not introduce spurious $\lambda$-shifts between the calibration lamp and the stellar lines. We have observed $\lambda 6717.69$ in the lunar spectrum, and the line center is within $0.01 \AA$ of the expected position. The displacement of atmospheric features from the D-lines, and the variation in $v_{r}$ for the

TABLE 1

Basic Data For The Program StaRs

\begin{tabular}{|c|c|c|c|c|c|c|c|}
\hline Star & $\begin{array}{l}\text { Spectral } \\
\text { Type }\end{array}$ & $m v^{*}$ & $\begin{array}{c}v_{r}(\text { cor }) \\
{\left[\mathrm{km} \mathrm{s}^{-1}\right]}\end{array}$ & $\left({ }^{(} \mathrm{Li} /{ }^{\prime} \mathbf{L i}\right)_{\mathbf{H}} \dagger$ & $n_{s}$ & $\begin{array}{c}v_{\text {rot }} \\
{\left[\mathrm{km} \mathrm{s}^{-1}\right]}\end{array}$ & $\lambda_{1 / 2}-6707.00 \AA$ \\
\hline $\begin{array}{l}\gamma \text { Lep } . \\
\xi \text { Peg. } \\
10 \text { Tau } \\
\chi \text { Her } \\
\chi^{1} \text { Ori } \\
\xi \text { UMa A } \\
\beta \text { Com. } \\
\delta \text { Tri } \\
\iota \text { Per . } \\
\kappa \text { Cet. . } \\
\xi \text { Boo A . } \\
\eta \text { Psc. . . } \\
\psi \text { UMa. . . } \\
\eta \text { Cet.. }\end{array}$ & $\begin{array}{l}\text { F6 V } \\
\text { F7 V } \\
\text { F8 V } \\
\text { F9 V } \\
\text { G0 V } \\
\text { G0 V } \\
\text { G0 V } \\
\text { G0 V } \\
\text { G0 V } \\
\text { G5 V } \\
\text { G8 V } \\
\text { G8 III } \\
\text { K1 III } \\
\text { K2 III }\end{array}$ & $\begin{array}{l}3.6 \\
4.2 \\
4.3 \\
4.6 \\
4.6 \\
4.4 \\
4.3 \\
4.9 \\
40 \\
4.8 \\
4.5 \\
3.7 \\
3.2 \\
3.4\end{array}$ & $\begin{array}{l}-9 \\
-5 \\
+29 \\
+52 \\
-13 \\
-17 \\
+5 \\
-6 \\
+51 \\
+20 \\
+2 \\
+15 \\
-19 \\
+12\end{array}$ & $\begin{array}{r}0.04 \\
0.01 \\
0.55 \\
0.03 \\
-0.01 \\
0.21 \\
0.39 \\
0.52 \\
0.32 \\
039 \\
-0.08 \\
. \\
.\end{array}$ & $\begin{array}{r}15 \\
10 \\
17 \\
11 \\
23 \\
20 \\
21 \\
3 \\
12 \\
12 \\
6 \\
18 \\
2 \\
13 \\
10\end{array}$ & $\begin{array}{r}9 \\
9 \\
7 \\
5 \\
10 \\
4 \\
5 \\
\leq 6 \\
6 \\
5 \\
5 \\
\leq 4 \\
3 \\
3\end{array}$ & $\begin{array}{l}082 \\
0.82 \\
0.82 \\
0.81 \\
0.83 \\
0.81 \\
0.81 \\
0 \quad 78 \\
0.80 \\
0.80 \\
0.81 \\
0.81 \\
0.79 \\
081\end{array}$ \\
\hline
\end{tabular}

* Hoffleit (1964)

† Herbig (1964) 
spectroscopic binary $\xi \mathrm{UMa} A$ as compared with the orbit of van den Bos (1927), combined with the results for the lunar spectrum and the separation of the components of the $\mathrm{Li}$ resonance line in scans of the calibration lamp, give us confidence that the instrument does not introduce spurious shifts in $(s, P)$ between the calibration lines and the stellàr lines. Hence, our wavelength scale for the stellar Li line is correct.

The observational procedure consisted of making from three to five consecutive scans of the $\mathrm{Li}$ and $\mathrm{Ca}$ lines for a star, accumulating about 800 counts per channel per scan. Points in the scans were $0.032 \AA$, apart. These were averaged, and then the average profiles of each line for each night of a particular run were averaged with any corrections necessary for changes in the solar correction to $v_{r}$ or in the calibration $\left(s_{0}, P_{0}\right)$. Calibration measurements were made several times per night for both $\lambda 6707.76(\mathrm{Li} \mathrm{I})$ and $\lambda 6717.04(\mathrm{Ne} \mathrm{I})$, which was more than adequate to monitor the slight variations in $\left(s_{0}, P_{0}\right)$ that do occur, probably as a consequence of temperature changes. Final line profiles were made for all stars except the spectroscopic binaries by averaging the averages for each run with the above corrections. These corrections are necessary so that channels of the same wavelength in the rest frame of the star are averaged. Photon noise plus residual uncompensated seeing fluctuations are such that an average of 10 scans gives a line profile with better than 2 percent accuracy for each point.

In Table 1 the program stars, spectral types, apparent visual magnitudes, corrected radial velocities, apparent isotope ratios measured by Herbig (1964), number of scans of the resonance line of $\mathrm{Li}$ I that were made, rotational velocities, and $\lambda_{1 / 2}$ (the last two of which are described later) are listed. Care was taken to ensure that there was no contamination by companions in binary systems. When necessary, the image rotator was used to keep the secondary out of the entrance aperture.

An upper limit to the parasitic light present in the scans can be evaluated from measurements of the instrumental response to a single line in the calibration lamp (such as $\lambda 6717.04$ of $\mathrm{Ne} \mathrm{I}$ ) over a whole spectral free range. If one assumes no continuum or weak lines in the lamp, it has been estimated in this fashion that in a star's spectrum, an infinitely sharp line would be filled by 13 percent by radiation beyond $0.12 \AA$ from the central maximum transmission. A feature as broad as $\lambda 6707$ would be filled by about 10 percent. A 13 percent correction to our observed depth of the $\mathrm{Ca}$ I feature at $6717.69 \AA$ in the lunar spectrum gives good agreement with the depth of the line in the Utrecht atlas (Minnaert, Mulders, and Houtgast 1940), and the equivalent width $W_{\lambda}$ of the $\mathrm{Ca} \mathrm{I}$ line when corrected for leakage agrees well with that given by Moore, Minnaert, and Houtgast (1966). When corrected for parasitic light, $W_{\lambda}$ from interferometric profiles of stellar $\mathrm{Li}$ and $\mathrm{Ca}$ lines agree well with those measured by the author from highdispersion (4.1 and $6.7 \AA \mathrm{mm}^{-1}$ ) photographic spectra.

\section{THEORETICAL LINE PROFILES}

Theoretical line profiles for the $\mathrm{Li}$ resonance line have been computed for various $\mathrm{Li}$ abundances and isotope ratios. A line absorption coefficient was used at each depth and frequency point which is the sum of that for each of the four components of the line. Each component has an absorption coefficient which is assumed to be a Voigt function. This summed line absorption coefficient is integrated, assuming pure absorption, through model atmospheres generated by the program AtLAS, written by S. E. Strom and R. L. Kurucz. Theoretical profiles are obtained from models of varying effective temperature (denoted $T_{\text {eff }}$ ). In Figure 1, profiles for a constant ${ }^{7} \mathrm{Li}$ abundance and values of ${ }^{6} \mathrm{Li} /{ }^{7} \mathrm{Li}$ of $0.0,0.25,0.50$, and 1.00 are shown for the model with $T_{\text {eff }}=6250^{\circ} \mathrm{K}$. (All models have $\log$ [surface gravity] $=4.0$.)

These theoretical profiles must be convoluted with the instrumental profile as determined from measurements of profiles for the sharp lines in the hollow-cathode lamp. Furthermore, they must be convoluted with profiles for line broadening due to rotation and also corrected for parasitic light before the observed and calculated profiles can be 


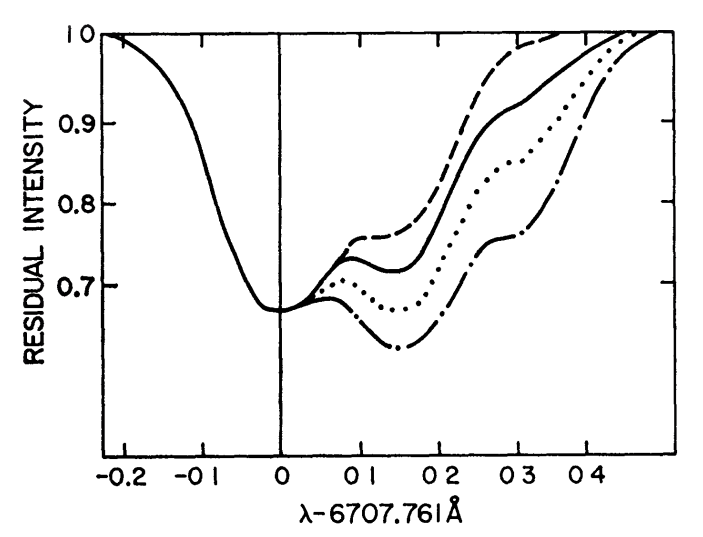

Fig. 1.-Calculated theoretical profiles for the resonance line of $\mathrm{Li}$. The ${ }^{7} \mathrm{Li}$ abundance is constant, while for the dashed line ${ }^{6} \mathrm{Li} /{ }^{7} \mathrm{Li}=0.0$, for the solid line it is 0.25 , for the dotted line 0.50 , and for the dot-dash line it is 1.00 . The model used to generate these profiles had $T_{\text {eff }}=6250^{\circ} \mathrm{K}$.

compared. The $\mathrm{Li}$ resonance line, because of its composite nature and large intrinsic width, is not very sensitive to the choice of rotational velocity (denoted $v_{\text {rot }}$ ), while the $\mathrm{Ca}$ I feature at $6717.69 \AA$, which is a single line, is much more sensitive to $v_{\text {rot }}$.

For each star, $T_{\text {eff }}$ is derived from the spectral type. The microturbulent velocity $v_{t}$, which is large enough that we can reproduce the observed $W_{\lambda}$ of the $\mathrm{Ca}$ I feature without requiring the stars to have higher $\mathrm{Ca}$ abundances than the Sun, is then determined. (The line at $6717.69 \AA$ is on the flat part of the curve of growth.) This requirement yields that $v_{t}$ must be greater than zero, and close to the solar value, for all stars except $\chi^{1}$ Ori and 10 Tau. For these two stars, $v_{t}$ must be at least $2.5 \mathrm{~km} \mathrm{~s}^{-1}$ to avoid a Ca overabundance of a factor of two or more. The Ca line profile for this choice of $v_{t}$ and model atmosphere is then convoluted with various rotational-broadening functions, and the best fit to the observed profile yields $v_{\text {rot }}$. Thus, $v_{t}$ is determined by $W_{\lambda}$ for $\lambda 6717.69$ of $\mathrm{Ca} \mathrm{I}$, and $v_{\text {rot }}$ from the width at half-maximum of the $\mathrm{Ca} \mathrm{I}$ line. With the chosen model, $v_{t}$, and $v_{\text {rot }}$, profiles for the $\mathrm{Li}$ resonance line are calculated to determine the $\mathrm{Li}$ abundance and isotope ratio. The calculated profile for $v_{t}=1.4 \mathrm{~km} \mathrm{~s}^{-1}$ and $v_{\text {rot }}=2 \mathrm{~km}$ $\mathrm{s}^{-1}$ for a model with $T_{\text {eff }}=6000^{\circ} \mathrm{K}$ fits the $\mathrm{Ca}$ line observed in the lunar spectrum, which is blended with a weak $\mathrm{Fe}$ I line at $6717.53 \AA$. The observed lunar profile is thus slightly asymmetric.

The rotational velocities deduced from this fitting procedure are listed in Table 1. Since the Ca I line $\lambda 6717.69$ becomes too strong in the last three stars of Table 1 to be fitted by a theoretical profile assuming pure absorption, $v_{\text {rot }}$ for these stars was determined from the profile of $\lambda 6710.32$ of $\mathrm{Fe}$. This line was observed with an etalon with a smaller free range and full width of the instrumental profile at half-maximum of $0.058 \AA$. The difference in the profile of the $\mathrm{Ca}$ I or $\mathrm{Fe}$ I line between $v_{\text {rot }}=0$ and $v_{\text {rot }} \geq 3 \mathrm{~km} \mathrm{~s}^{-1}$ is noticeable. The rotational velocities have an estimated accuracy of \pm 20 percent. The agreement between our values of $v_{\text {rot }}$ and those of Kraft (1967) for the four cases we have in common is good. It is somewhat surprising that none of the stars had lines as sharp as the lunar spectrum. This may be related to the unusually large number of stars with $\mathrm{H}-\mathrm{K}$ emission present in our program group compared with the percentages given by Wilson (1963) of randomly selected field stars of various spectral types showing $\mathrm{H}-\mathrm{K}$ emission.

\section{INTERPRETATION OF THE LITHIUM PROFILES}

Two methods can be used to determine the $\mathrm{Li}$ isotope ratio. One of these relies on the center-of-gravity wavelength $\left(\lambda_{1 / 2}\right)$ of the line, such that the equivalent width of the line on each side of $\lambda_{1 / 2}$ is the same. With our theoretical profiles we have verified the 
accuracy of the relationship between $\lambda_{1 / 2}$ and ${ }^{6} \mathrm{Li} /{ }^{7} \mathrm{Li}$ which was first given by Herbig (1964), namely,

$$
\lambda_{1 / 2}=\frac{6707.811+\left({ }^{6} \mathrm{Li} /{ }^{7} \mathrm{Li}\right) 6707.971}{1+\left({ }^{6} \mathrm{Li} /{ }^{7} \mathrm{Li}\right)} \AA .
$$

Herbig (1964) attempted to measure $\lambda_{1 / 2}$ for $\lambda 6708 \AA$ from photographic spectra whose resolution was inadequate to yield profiles of the $\mathrm{Li}$ line. Herbig's original spectra were remeasured by the author with an oscilloscope Grant machine to obtain results consistent with the original determinations of the wavelengths of the $\mathrm{Li}$ feature in various field stars. However, when measurements were made on a number of additional spectra at $6.7 \AA \mathrm{mm}^{-1}$ taken by the author with the 100-inch telescope on Mount Wilson, using the new 09801 emulsion, it became apparent that the value of $\lambda_{1 / 2}$ measured with photographic spectra was not reproducible from one spectrogram to another of the same star. Herbig's original determination apparently suffered from too small a number of spectra/ star and grain effects.

Interferometric profiles of $\lambda 6708 \AA$ were planimetered to yield $\lambda_{1 / 2}$, with the wavelength scale determined by the corrected $v_{r}$, and hence the $\mathrm{Li}$ isotope ratio. The measured values of $\lambda_{1 / 2}$ are listed in the last column of Table 1 . Note that $\lambda_{1 / 2}=6707.81 \AA$ for ${ }^{6} \mathrm{Li} /{ }^{7} \mathrm{Li}=0.0$. This method, however, depends on the wavelength scale and thus reflects any residual errors in the corrected $v_{r}$. The isotope ratio can also be determined from the theoretical fits to the observed resonance-line profile, where the isotope ratio essentially affects the width at half-maximum depth of the $\mathrm{Li}$ line by changing the strength of the long-wavelength wing of the feature. This second method of deriving ${ }^{6} \mathrm{Li} /{ }^{7} \mathrm{Li}$ is independent of any errors in $v_{r}$, but does require an accurate $v_{\text {rot }}$ and $v_{t}$. For rapidly rotating stars, the line profile becomes symmetric about a wavelength which depends on ${ }^{6} \mathrm{Li} /{ }^{7} \mathrm{Li}$, and the second method will not work well in these cases.

In Figure 2 the observed and calculated $\mathrm{Li}$ profiles in eight of the stars, and the $\mathrm{Ca} \mathrm{I}$ profile for one of the stars, are shown. The observed points are separated by $0.032 \AA$. The position of 6707.761 or $6717.69 \AA$ determined by the corrected $v_{r}$ is indicated by the subscript "o," while the wavelength scale of the theoretical profile is indicated by the subscript "p." The theoretical profiles are calculated with the values of $v_{t}$ and $v_{\text {rot }}$ for each star discussed in $\S$ III. The observed profiles have been corrected for dark count, but not for parasitic light. A correction for parasitic light of 10 and 13 percent of the continuum for $\mathrm{Li}$ and $\mathrm{Ca}$ lines, respectively, was applied to the theoretical profiles. All the calculated profiles for the $\mathrm{Li}$ resonance line have ${ }^{6} \mathrm{Li} /{ }^{7} \mathrm{Li}=0.0$. Note the good agreement (with the possible exception of $\chi^{1}$ Ori) between the two wavelength scales, and also between the calculated and observed line profiles. In no case is a ratio of ${ }^{6} \mathrm{Li} /{ }^{7} \mathrm{Li}$ significantly greater than 0.0 indicated.

The final values of ${ }^{6} \mathrm{Li} /{ }^{7} \mathrm{Li}$ derived by these two methods are $0.1( \pm 0.1)$ for $\chi^{1}$ Ori, $0.05( \pm 0.1)$ for $\gamma$ Lep, $\xi$ Peg, and $10 \mathrm{Tau}$, and $0.0( \pm 0.1)$ for the rest of the stars. The isotope ratio could be $0.0( \pm 0.1)$ for all the stars, and the special difficulties for relatively rapidly rotating stars $\left(v_{\text {rot }} \geq 7 \mathrm{~km} \mathrm{~s}^{-1}\right)$ could introduce the apparent cases of ${ }^{6} \mathrm{Li} /{ }^{7} \mathrm{Li}$ greater than zero.

\section{CONCLUSIONS}

The accurate values of $v_{\text {rot }}$ which we have obtained for these slowly rotating stars, and the good agreement with the scale of Kraft (1967), are of some interest. The principal result is the low values of ${ }^{6} \mathrm{Li} /{ }^{7} \mathrm{Li}(\leq 0.1)$ that we have obtained by $\mathrm{FP}$ interferometry, which remove the discrepancy between predictions of the relative destruction rates of ${ }^{6} \mathrm{Li},{ }^{7} \mathrm{Li}$, and $\mathrm{Be}$, and the previously observed values (see the review of Wallerstein and Conti 1969). With the lower isotope ratios, the theoretical predictions for ${ }^{6} \mathrm{Li} /{ }^{7} \mathrm{Li}$ as a function of $\mathrm{Li}$ and $\mathrm{Be}$ depletion are obeyed. As a by-product of the calculation of theoretical profiles, we have verified previous assumptions for $W_{\lambda}$ and $\lambda_{1 / 2}$ of the $\mathrm{Li}$ resonance line, at least for cases where $W_{\lambda}$ of $\lambda 6708 \AA$ is less than $150 \mathrm{~m} \AA$. Thus, 


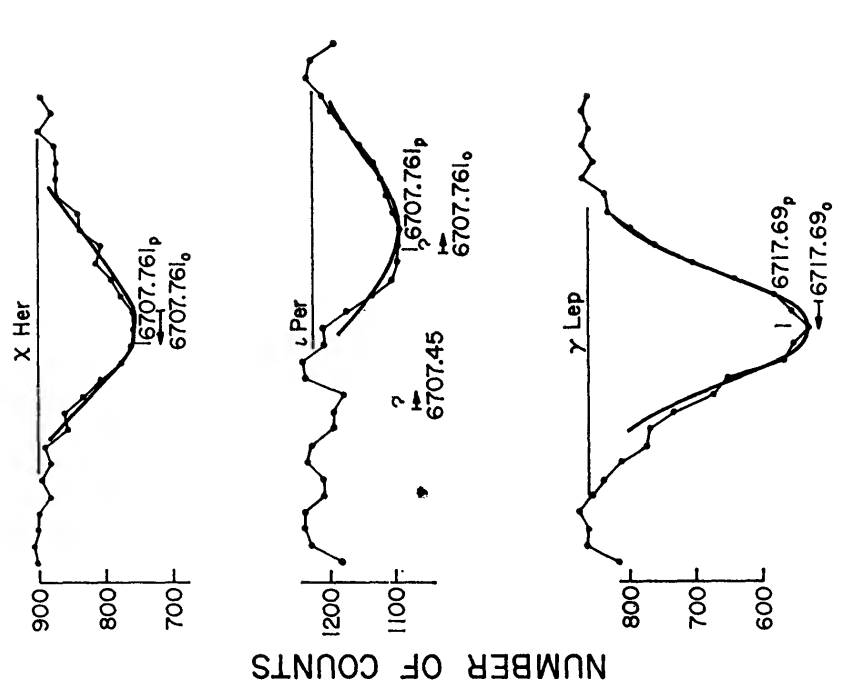

ธิษ)

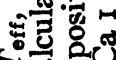
of 焉点
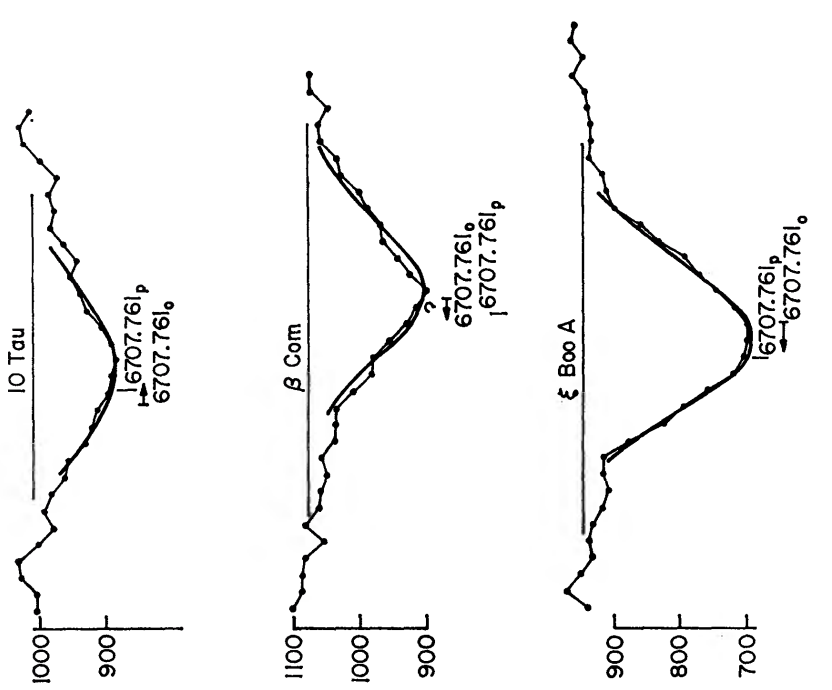

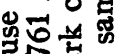

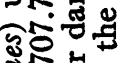
今응

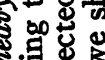

잉

政

ac

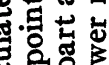

政

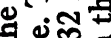

.

巴刃 호

की

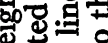

政要

4 政:

ت도은

당

贯啳

运

邹

s.

:

.

吾

SINกOJ to y YGWNN
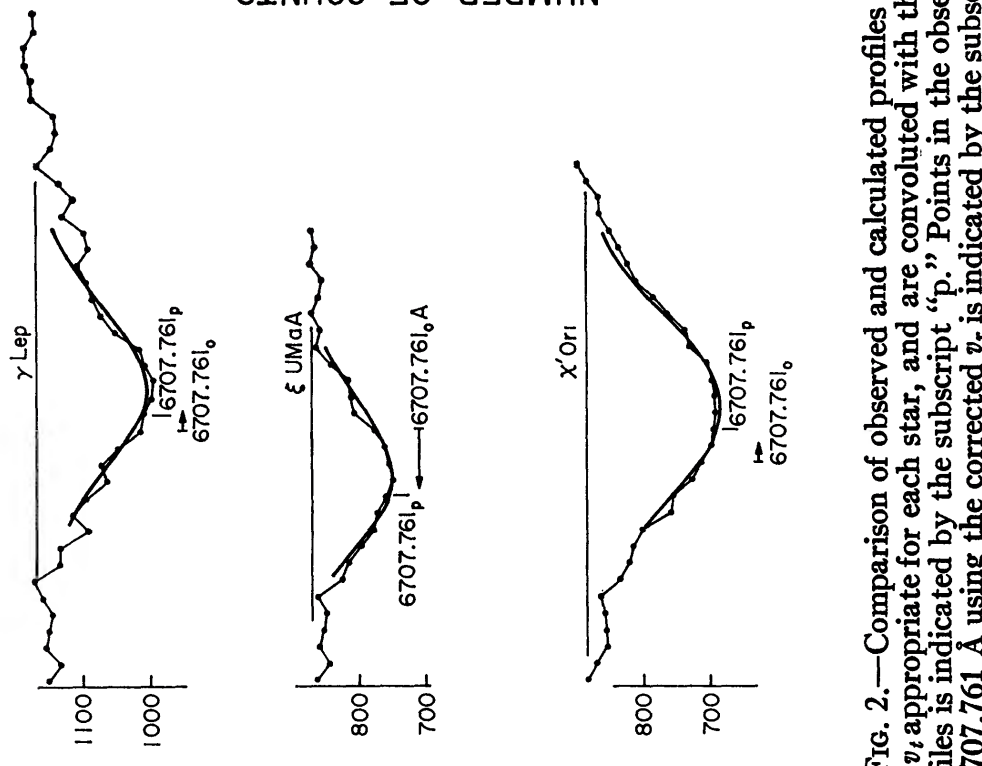

定要

政.
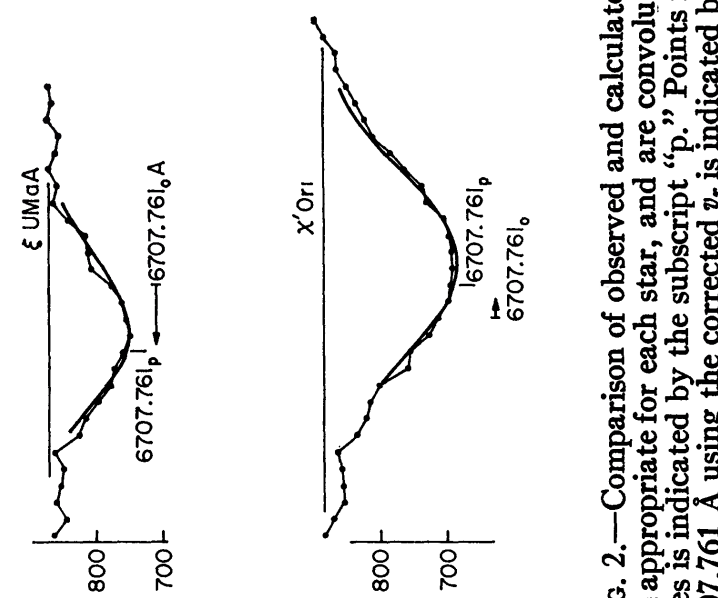

उ० 0 .

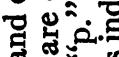

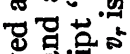

要

路家

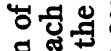

울

年

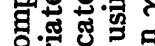

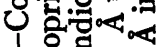

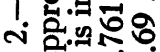

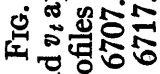

SLNกOJ tO Y 
the previous $\mathrm{Li}$ abundance determinations are correct to within small factors. Furthermore, we have compared the strength of the subordinate Li line (at $6103 \AA$ ) in T Sgr $(\mathrm{S} 5,8 \mathrm{e})$ with a nearby $\mathrm{Ca}$ I line with the same excitation potential to obtain a $\mathrm{Li}$ abundance which is approximately that obtained by Boesgaard (1970). Thus the enormous $\mathrm{Li}$ overabundances previously derived for some of the $\mathrm{C}$ and $\mathrm{S}$ stars are correct.

I would like to thank Professor Guido Münch, who supervised this research, which was part of my doctoral thesis, and provided much assistance in making the observations. I thank Professor G. Herbig for the loan of his spectra and valuable conversations. I am grateful to Mr. Kurucz and Dr. Strom for the use of their computer programs. Financial support from the California Institute of Technology is gratefully acknowledged.

\section{REFERENCES}

Boesgaard, A. M. 1970, Ap. J., 161, 83.

Bos, W. H. van den. 1927, Danske Videnskabernes Selskab, 12, 295.

Conti, P. S. $1969, A p . J$. (Letters), 155, L167.

Feast, M. W. 1970, M.N.R.A.S., 148, 489.

Herbig, G. H. 1964, A p.J., 140, 702.

Hoffleit, D. 1964, Yale Catalogue of Bright Stars (New Haven: Yale University Press).

Kraft, R. P. 1967, Ap.J., 150, 551.

Krankowsky, D., and Müller, O. 1969, Geochim. Cosmochim. Acta, 31, 1833.

Minnaert, M., Mulders, V. F. W., and Houtgast, J. 1940, Photometric Atlas of the Solar Spectrum (Utrecht: Sterrewacht Sonnenborgh).

Moore, C. E., Minnaert, M. G. J., and Houtgast, J. 1966, N.B.S. Monog., No. 61.

Vaughan, A. H. 1967, Ann. Rev. Astr. and Ap., 5, 139.

Wallerstein, G., and Conti, P. S. 1969, Ann. Rev. Astr. and Ap., 7, 99.

Wilson, O. C. 1963, Ap.J., 138, 832.

- 1968, ibid., 153, 221. 
